\title{
Editorial
}

\author{
APPLICATION OF MONOCLONAL ANTIBODIES \\ TOWARDS IMMUNOLOGICAL STUDIES IN \\ LEPROSY
}

Immunology has a key role in the study of leprosy. Unlike any other infectious disease, the immune system decides not only whether the outcome from contact with the infection will lead to protection (self-healing) or disease, but also dictates the pattern of clinical manifestations. The clinical classification of forms of leprosy has been based fundamentally on shifting balance between T-cellmediated and humoral reactions to Mycobacterium leprae. ${ }^{1}$ This classification has been widely accepted apparently as the best guidance to the two polar or borderline types of the disease. However, it is surprising that these investigations often refer to the response of the immune system to $M$. leprae in toto, with only scant attention to the fact that diverse reactions may arise towards the antigenic determinants (i.e. epitopes) of the various protein, glycolipid and polysaccharide constituents $^{2}$ of the leprosy bacillus. Since lymphocytes react towards these epitopes of the bacillus individually, it seems conceivable that those immune reactions which play a critical role for the pathogenesis of leprosy would not be random but rather restricted in specificity to certain structures of the leprosy bacillus.

Progress in the analysis of the specificity of cell-mediated and humoral immunity in leprosy has been slow and further advance could be expected only from the definition and purification of the relevant antigens. The purpose of the subsequent discussion will be to bring attention to a novel technology, i.e. hybridoma cell line derived monoclonal antibodies (MAB), which could greatly contribute towards the identification of those antigens which are functionally important for the development and progression of the respective forms of the disease.

\section{General principles and technology}

Antisera from immunized individuals represent 'polyclonal antibodies', as they 
are derived from multiple clones of $\mathrm{B}$ cells, each responding to the individual epitopes of Mycobacterium leprae and even heterogeneous in response to one epitope in terms of affinity and isotype. Monoclonal antibodies are uniform in specificity and structure, being the product of a single B-lymphocyte clone which has been 'immortalized' by fusion with an autonomously growing myeloma cell. The technology has been well described in various excellent reviews and monographs. ${ }^{3,4}$ Briefly, spleen lymphocytes harvested from immunized mice are fused in the presence of polyethyleneglycol with myeloma cells which are deficient in the enzyme hypoxanthine guanine phosphoribosyl transferase (HPRT). Deficiency in this enzyme of the 'salvage pathway' of nucleic acid synthesis will selectively prevent myeloma cells from growing in selective culture media (HAT) containing aminopterin which blocks the main biosynthetic pathway of nucleic acids whilst allowing the growth of hybrid cells which had acquired HPR T from the lymphocyte fusion partner.

The actual fusion manipulation takes less than $1 \mathrm{~h}$ to perform. The cells distributed at $10^{5}-10^{6}$ per well frequency in plastic trays will manifest macroscopically discernible colony growth within 7-14 days. At this stage, culture media are tested for the presence of antibodies by a sensitive test, e.g. radioimmunoassay and the cells from antibody-positive wells are cloned by limiting cell dilution. Stable viable cell lines, usually of lower yield than the number of positive primary wells, are stored frozen in liquid nitrogen and grown as ascitic tumour in mice which generates ascites fluid for bulk production of the monoclonal antibody.

\section{Review of reported MABs to Mycobacterium leprae}

The salient characteristics of MABs described in recent publications ${ }^{5-7}$ are summarized in Table 1. Although only one representative MAB clone for each specificity is listed here, several other hybridomas of apparently overlapping specificity were generated at least for the M. leprae-specific MYla and MY2a specificities. ${ }^{6}$ The specificity of MABs was designated by prefix MY (mycobacterial) and an arbitrary number, defining the distinct molecules (e.g. MY1, MY2, etc.); the letters distinguish between the epitopes expressed on the same molecule (e.g. MY4a, MY4b). It would be beneficial upon agreement with other investigators to compare reagents and to introduce a common nomenclature. The $68 \mathrm{Kd}$ molecule carries at least one identified species-specific (IVD8) and one cross-reactive (IIH9) epitope. ${ }^{5}$ Similarly, it is likely that MY 1 (12Kd) and MY2 antigens would carry in addition to their species specific epitopes other determinants possibly of the cross-reactive type. It is of interest, that all anti Mla and M2a hybridoma lines were generated from spleens of mice which had been immunized with the supernatant fraction of sonicated $M$. leprae, whilst spleens from whole sonicate injected mice yielded only cross-reactive MABs. ${ }^{6}$ Conceiv- 
Table 1. Review of monoclonal antibodies to Mycobacterium leprae

\begin{tabular}{|c|c|c|c|c|}
\hline $\begin{array}{l}\text { Monoclonal antibody } \\
\text { code/specificity-class }\end{array}$ & $\begin{array}{l}\text { Chemical structure- } \\
\text { molecular weight }\end{array}$ & Localization & $\begin{array}{l}\text { Cross- } \\
\text { reactivity }\end{array}$ & Ref. \\
\hline ML06/MYla-IgGl & Protein-12Kd & Cytoplasm & None & 6 \\
\hline ML04/MY2a-IgGl & Protein & Cytoplasm(?) & Marginal§ & 6 \\
\hline ML30/MY3a-IgG1 & Protein-35-70Kd $\dagger$ & Cell wall & Broad & 6 \\
\hline $\begin{array}{l}\text { ML02/MY4a-IgG3* } \\
\text { ML34/MY4b-IgM* }\end{array}$ & Polysaccharide- $40-50 \mathrm{Kd} \ddagger$ & Cell wall & $\begin{array}{l}\text { Broad } \\
\text { Broad }\end{array}$ & $\begin{array}{l}6 \\
6\end{array}$ \\
\hline $\begin{array}{l}\text { IVD8-IgG1 } \\
\text { IIH9-IgG1 }\end{array}$ & Protein-68Kd & Cytoplasm & $\begin{array}{l}\text { None } \\
\text { Broad }\end{array}$ & $\begin{array}{l}5+\dagger \\
5\end{array}$ \\
\hline $\begin{array}{l}\mathrm{PG}_{2} \mathrm{~B} 8 \mathrm{~F}-\mathrm{IgM} \\
\mathrm{AM}-8-2 \mathrm{C} 2-\mathrm{IgM}\end{array}$ & $\begin{array}{l}\text { Glycolipid } \\
\text { Arabinomannan }\end{array}$ & $\begin{array}{l}\text { Cell wall } \\
\text { Cell wall }\end{array}$ & $\begin{array}{l}\text { Marginal** } \\
\text { All mycobacteria }\end{array}$ & $\begin{array}{l}5+\dagger \\
5+\dagger\end{array}$ \\
\hline A-494-IgM & $?$ & Cell wall & All mycobacteria & 7 \\
\hline
\end{tabular}

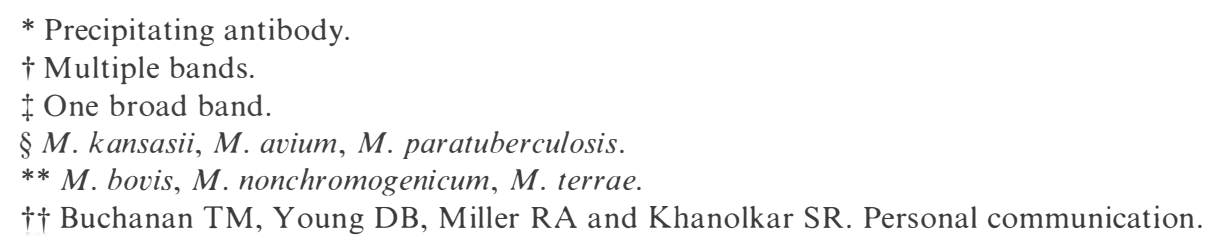

ably, the presence of cell-wall constituents could have suppressed the murine antibody response to the species-specific protein antigens.

\section{TAXONOMIC ASPECTS}

It may seem disappointing that none of the MABs can 'type' for an antigen which would distinguish between slow and fast growing species of mycobacteria. Neither is there a linkage with any of the defined biochemical markers. The two $M$. leprae-specific epitopes, MYla and MY2a are not expressed by mycobacteria strains ICRF or W which have been implicated for closer relationship with $M$. leprae, but their presence in "leprosy-derived corynebacteria" ${ }^{8}$ is yet to be determined.

It is of interest to characterize the antigens with epitopes which are shared between $M$. leprae and the cultivable species of mycobacteria. Antibodies of restricted cross-reactivity are of best use here. ${ }^{6}$ The MY2a epitope is marginally represented only on 3 other species, namely $M$. kansasii, $M$. avium and $M$. paratuberculosis. The MY3a epitope varies quantitatively between several species and may be of interest for experimental models since it is expressed strongly on $M$. lepraemurium. The best 'typing' pattern is demonstrable with MABs directed towards the two distinct epitopes of the MY4 polysaccharide antigen which segregate in several species of mycobacteria almost in a reciprocal manner. The 
MY 4a epitope is most pronounced on mycobacterium strain W and also on $M$. avium and $M$. kansasii whereas MY4b has the strongest expression on $M$. tuberculosis, $M$. bovis, $M$. scrofulaceum and $M$. paratuberculosis. The relevance of these relationships and their possible utility in immunization programmes is yet to be explored. Hence, it may be worthwhile to determine the immune response to a shared epitope of $M$. leprae when presented in the context of another mycobacterial species.

\section{Antigen deposits in tissues}

Probably the most ominous outcome of anergy in lepromatous patients relates to the impaired clearance of Mycobacterium leprae bacilli. Although the histological localization and amount of whole or fragmented bacilli have been examined previously, little is known about the composition of the retained antigens in tissues. Nevertheless, this may be important, since glycolipids have been implicated in the induction of granulomas. Immunohistological studies using enzyme-labelled MABs binding to distinct antigens are now technically attainable. This approach should be superior to acid-fast staining which may no longer detect certain constituents or to staining with polyclonal antisera which do not discriminate between the multitude of antigens. It would be of particular interest to analyse the antigenic composition in patients with tuberculoid or indeterminate leprosy where the deposits are infrequent and small. However, the paucity of bacilli does not exclude the possible role of a particular mycobacterial antigen in situ. The role of deposited antigen or immune complexes has been implicated for the pathogenesis of erythema nodosum leprosum. If the clinical manifestations result from 'slow degradation of large amounts of antigen's it would be pertinent to identify the constituents which are most resilient to breakdown and possibly with biological (e.g. adjuvant) activity. This is relevant also to chemotherapy which may gradually eliminate viable bacilli but leave certain cell-wall constituents to persist for prolonged periods.

\section{Circulating immune complexes}

Their composition in sera of patients with lepromatous leprosy has been analysed recently. ${ }^{10}$ The results indicated that the number of mycobacterial constituents in these complexes is rather restricted and so far only one protein antigen with a molecular weight of about $67 \mathrm{Kd}$ has been identified. Monoclonal antibodies directed towards two distinct epitopes of an antigen of corresponding molecular weight have been described ${ }^{5}$ and if their binding to complexed antigen is established, they could be valuable specific tools for monitoring the levels of circulating complexes. 


\section{Serological assays for leprosy}

Antibody levels towards antigens of Mycobacterium leprae have been studied extensively by several previous investigators who found that they become elevated consistently in patients at the lepromatous end of the clinical spectrum. It was considered that antibody levels may help to monitor the bacterial load or may predict the possibility of a relapse during treatment. However, the employed serological tests were more or less lacking in specificity. A novel serological test based on the use of species-specific monoclonal antibodies has been developed recently. ${ }^{1}$ In this assay, the binding of a radio- or enzyme-labelled MAB (ML04) to wells of microtiter plates which had been precoated with $M$. leprae soluble antigen is competitively inhibited by serially diluted sera from patients. Thus, only those human antibodies which have specificity matching the combining site of the labelled MAB probe would give positive inhibitory values. Protein molecules from $M$. leprae may carry both species-specific and cross-reactive epitopes which in turn would break down the specificity of a direct antibody binding test even if the antigen was purified to homogeneity. This problem does not affect the serum competition test in which the epitope specificity is safeguarded by a selected specific MAB. Using this test, almost all tested LL patients showed ML04 binding inhibitory antibody levels. " Preliminary results have shown demonstrable antibody levels also in a certain proportion of tuberculoid patients and even in healthy family contacts of leprosy patients (Sinha and Sengupta, in preparation). These pilot studies are encouraging and require further evaluation in long-term prospective clinical trials. The merit of the test could be prognostic, whereby an increase in antibody levels would indicate the shifting of the disease towards the lepromatous pole of the spectrum. However, epidemiologically the most valuable outcome would be if the test, on the strength of its specificity, would differentiate (a) between infected and non-infected healthy subjects; or (b) predict on a prognosis for self-healing or progressive disease in endemic areas.

\section{Potentials for a Mycobacterium leprae specific skin test and lymphocyte stimulation test (LST)}

The 24-72 h skin erythema and induration reaction to intracutaneous injection of the soluble fraction of sonicated $M$. leprae (i.e. Dharmendra's antigen, or leprosin) represents a $\mathrm{T}$-cell mediated, delayed type hypersensitivity reaction which reflects the response to previous $M$. leprae infection. Earlier attempts with the fractionation of these crude bacterial extracts met with only partial success.

For epidemiological and diagnostic purposes, it is clearly desirable to distinguish between previously $M$. leprae infected and non-infected, yet clinically healthy persons in both endemic areas. This seems feasible in the light of the 
report that non-exposed individuals with high LST responses to PPD showed an almost complete lack of 72-h skin reactions to the soluble fraction of sonicated $M$. leprae hence suggesting the existence of T-cell stimulating, $M$. leprae specific antigens. ${ }^{12}$ However, others reported that lymphocytes from the majority of non-exposed healthy subjects showed proliferative responses to soluble sonicated $M$. leprae antigen. ${ }^{13,14}$ Therefore, the diagnostic reagent to sustain an $M$. leprae specific test needs further purifications and structural studies. Since the T-cell responses to mycobacteria are mediated by protein molecules and not by the polysaccharide or glycolipid constituents, the MAB-defined MY1 or MY2 molecules when isolated and supplied in adequate quantities, appear to be possible candidates for a specific skin test. So far, we attempted in our laboratory the purification of MY 1 by MAB-based affinity chromatography and achieved about $10 \%$ yields of partially purified antigen. This material when tested in guinea-pigs showed cross-reactivity with PPD, but it was not possible to decide whether that was attributable to cross-reactivity of the MY1 molecule or of the contaminating antigens (R J W Rees, unpublished results).

Studies with other protein antigens have suggested that the epitopes recognized by $\mathrm{B}$ and $\mathrm{T}$ cells respectively are structurally distinct. Although the molecules which carry the $M$. leprae specific epitopes recognized by MABs remain the best candidates for the search for T-cell stimulatory structures, it may be necessary to carry out this analysis further at the level of peptide structure. ${ }^{15,16}$

\section{Analysis of T-cell anergy in lepromatous leprosy}

A controversy in this area relates to the degree of specificity. Anergy may be (i) clonally restricted to either Mycobacterium leprae specific ${ }^{17-21}$ or to common mycobacterial antigens, ${ }^{22}$ or (ii) a polyclonal defect in the T-cell immune system. The cellular mechanisms are considered to be either (a) deletion of the responding helper $\left(\mathrm{T}_{\mathrm{H}}\right)$ cells, ${ }^{19,21}$ or $(\mathrm{b})$ activation of suppressor $\left(\mathrm{T}_{\mathrm{S}}\right)$ cells. ${ }^{17,23}$

It has been demonstrated in experimental models that $T_{S}$ cells can be specific to one molecule of a cellular antigen or even specific to a particular epitope of a protein antigen. ${ }^{24}$ It would be feasible to determine whether any of the MAB-defined epitopes activate $\mathrm{T}_{\mathrm{S}}$ cells. Here, the primary interest in the $M$. leprae specific epitopes is supported by the results obtained by LST as well as by skin reactions that anergy is induced by $M$. leprae soluble antigen but not by PPD. ${ }^{18,19,21}$ Thus, $M$. leprae specific epitopes may be activating $\mathrm{T}_{\mathrm{S}}$ cells which effect directly ${ }^{17}$ or via macrophages ${ }^{23}$ the suppression of the mitogenic response to antigens represented in other species of mycobacteria and also the polyclonal response to Concanavalin $\mathrm{A}$. It is important to distinguish between the highly specific activation stage and the much less restricted effector stage of suppression. Interestingly, the macrophage lysate mediated suppression is not entirely 
non-specific, ${ }^{23}$ since suppression was most pronounced in response to $M$. kansasii and $M$. avium, both of which express, at least weakly, the MAB defined MY2a epitope.

Long-term dapsone therapy causes a reversion of T-cell anergy to common mycobacterial antigens but leaves a persistent suppression of the M. leprae specific response. ${ }^{25}$ This change in the specificity of anergy may be monitored as an appropriate parameter, possibly of prognostic value.

\section{Prophylactic immunization versus immunotherapy}

Acquired T-cell mediated immunity to mycobacterial infections is thought to confer protection against infections through activation of macrophages. However, it is understood that 'hypersensitivity' reactions as measured by the LST do not correlate with 'protective' immunity, since LST values are raised in many TT/BT cases with destructive disease whilst the values are negative or low in indeterminate patients, most of whom are self-healing. The failure of delayed type hypersensitivity to suppress mycobacterial growth was shown also in murine experiments. ${ }^{26}$ It has been speculated in leprosy (as in tuberculosis) that hypersensitivity and protective immunity may be directed towards separate antigens of mycobacteria. ${ }^{27}$ These thoughts were not ascertained experimentally presumably because of the lack of adequately purified antigens; nevertheless, the subject yet remains pertinent for study with MAB-defined antigens.

It may appear that prophylactic vaccination and immunotherapy, i.e. conversion of anergic lepromatous patients, represent different categories. However, a sharp differentiation may not be necessary when considering that potential anergy based on environmental or genetic grounds could represent the main problem also in the susceptible healthy individuals from endemic areas (a minority of the total population). Indeed, only a single rationale, embraced by the inoculation of live BCG with or without killed Mycobacterium leprae has so far been explored with partial success in prophylactic ${ }^{28}$ and 'therapeutic' immunizations ${ }^{29}$ as well as in murine experimental work.$^{30}$ Elucidation of the mechanisms of immunotherapy which is of key importance, may benefit from the use of molecularly defined antigens. Unlike other conventional vaccines, the optimal strategy for the pre-emption or reversion of active suppressor cells is yet to be defined.

\section{Synopsis}

Monoclonal antibodies produced by hybridoma cell lines are of restricted and uniform specificity. They represent reagents which are in many aspects superior to the heterogeneous mixture of 'polyclonal antibodies' present in antisera from immunized or infected individuals. The technology for producing MABs is now 
firmly established, relatively easy to perform and require facilities for tissue culture, immunoassays and a supply of an inbred strain of mice or rats. Three protein antigens (MY1-12K, MY2 and $68 \mathrm{~K}$ ) carrying distinct antigenic determinants, which are expressed by Mycobacterium leprae, but not by several other species of mycobacteria, have been identified by MABs. These $M$. leprae-specific determinants may help to make important advances in: (1) detection of antigen or immune complexes in tissues or body fluids; (2) serological diagnosis, disease monitoring and epidemiology; (3) development of a specific skin test; and (4) further research on therapeutic or prophylactic immunization against leprosy. So far, a serological test based on the use of the ML04 (anti-MY2a) monoclonal antibody has been developed and evaluated in a pilot study." The diagnostic potentials of this test as well as the various other possible applications of MABs deserve attention in future studies.

J IVANYI

\section{Department of Experimental Immunobiology}

The Wellcome Research Laboratories

Langley Court, Beckenham, Kent

\section{References}

${ }^{1}$ Ridley DS, Jopling WH. Classification of leprosy according to immunity. Int J Lepr, 1966; 34: 255.

2 Daniel TM, Janicki BW. Mycobacterial antigens: a review of their isolation, chemistry and immunological properties. Microbiol Rev, 1978; 42: 84.

${ }^{3}$ Galfre G, Milstein C. Preparation of monoclonal antibodies: strategies and procedures. Míthods in Enz ymology, 1975; 73: 3.

${ }^{4}$ Hammerling GJ, Hammerling U, Kearney JF. Monoclonal antibodies and T-cell hybridomas. Perspectives and technical advances. In Research Monographs in Immunology, Vol. 3. Turk JL (ed.), Elsevier/North-Holland, 1981.

${ }^{5}$ Gillis TP, Buchanan TM. Production and partial characterization of monoclonal antibodies to Mycobacterium leprae. Infection and Immunity, 1982; 37: 172.

${ }^{6}$ Ivanyi J, Sinha S, Aston R, Cussell D, Keen M, Sengupta U. Definition of species-specific and cross-reactive antigenic determinants of Mycobacterium leprae using monoclonal antibodies. Clin exp Imm, 1983; 52: 528.

7 Bach M-A, Hoffenbach A. A monoclonal antibody against Mycobacterium lepraemurium which recognizes a cross-reacting mycobacterial antigen. Ann Immunol (Inst Pasteur), 1983; 134C: 301.

8 Delville J, De Sloovere T, Fontaine F, Gueurm, M Ch, Rajjan W, Spina A, Cocito C. Biological properties of diphtheroid bacteria (LDC) isolated from human leprosy lesions. Acta Leprol, 1982; 88: 47.

9 Ridley MJ, Ridley DS. The immunopathology of erythema nodosum leprosum: the role of extravascular complexes. Lepr Rev, 1983; 54: 95.

${ }^{10}$ Chakrabarty AK, Maire M, Saha K, Lambert P-H. Identification of components of IC purified from human sera. II. Demonstration of mycobacterial antigens in immune complexes isolated from sera of lepromatous patients. Clin exp Imm, 1983; 51: 225. 
1 Sinha S, Sengupta U, Ramu G, Ivanyi J. A serological test for leprosy based on competitive inhibition of monoclonal antibody binding to the MY2a determinant of M. leprae. Trans Roy Soc Trop Med Hyg, 1983; 77: 869.

12 Smelt AHM, Rees RJW, Liew FY. Induction of delayed-type hypersensitivity to M ycobacterium leprae in healthy individuals. Clin exp Imm, 1981; 44: 501.

13 Bahr GM, Rook GAW, Stanford JL, Lydyard PM, Bryceson ADM. The effect of delayed addition of antigen and ' $E$ ' rosetting on the proliferative response to mycobacterial antigens of peripheral blood lymphocytes from normal individuals or from patients with tuberculosis or leprosy. Immunology, 1981; 44: 585.

14 Closs O. In vitro lymphocyte response to purified protein derivative, BCG and Mycobacterium leprae in a population not exposed to leprosy. Infect Immun, 1975; 11: 1163.

15 Lamb JR, Eckels DD, Lake P, Woody JN, Green N. Human T-cell clones recognize chemically synthesized peptides of influenza haemagglutinin. Nature, 1982; 300: 66.

16 Savrda J. Synthesis and biological assays of peptides from a tuberculin-active protein. Infect Immun, 1983; 40: 1163.

17 Mehra V, Mason LH, Fields JP, Bloom BR. Lepromin-induced suppressor cells in patients with leprosy. J Immunol, 1979; 123: 1813.

18 Haregewoin A, Godal T, Mustafa AS, Belehu A, Yemaneberhan T. T-cell conditioned media reverse T-cell unresponsiveness in lepromatous leprosy. Nature, 1983; 303: 342.

19 Stoner GL, Mshana RN, Touw J, Belehu A. Studies on the defect in cell-mediated immunity in lepromatous leprosy using HLA-D-Identical siblings. Scand J Immunol, 1982; 15: 33.

${ }^{20}$ Stanford JL, Nye PM, Rook GAW, Samuel N, Fairbank A. A preliminary investigation of the responsiveness or otherwise of patients and staff of a leprosy hospital to groups of shared or species-specific antigens of mycobacteria. Lepr Rev, 1981; 52: 321.

${ }^{21}$ Godal T, Myklestad B, Samuel DR, Myrvang B. Characterization of the cellular immune defect in lepromatous leprosy: a specific lack of circulating Mycobacterium leprae-reactive lymphocytes. Clin exp Imm, 1971; 9: 821.

22 Closs O, Reitan LJ, Negassi K, Harboe M, Belehu A. In vitro stimulation of lymphocytes in leprosy patients, healthy contacts of leprosy patients, and subjects not exposed to leprosy. Scand J Immunol, 1982; 16: 103.

${ }^{23}$ Salgame PR, Mahadevan PR, Antia NH. Mechanism of immunosuppression in leprosy: presence of suppressor factor(s) from macrophages of lepromatous patients. Infect Immun, 1983; 40: 1119.

${ }^{24}$ Sercarz EE, Yowell RL, Turkin D, Miller A, Araneo BA, Adorini L. Different functional specificity repertoires for suppressor and helper T cells. Immunological Rev, 1978; 39: 108.

${ }^{25}$ Reitan LJ, Closs O, Belehu A. In vitro lymphocyte stimulation in patients with lepromatous and borderline tuberculoid leprosy. The effect of dapsone treatment on the response to Mycobacterium leprae antigens, tuberculin purified protein derivative and non-mycobacterial stimulants. Int J Lepr, 1982; 50: 455.

${ }^{26}$ Lovik M, Closs O. Repeated delayed-type hypersensitivity reactions against Mycobacterium lepraemurium antigens at the infection site do not affect bacillary multiplication in $\mathrm{C} 3 \mathrm{H}$ mice. Infect Immun, 1982; 36: 768.

27 Ridley DS. Hypersensitivity and immunity reactions and classification. Lepr Rev, 1976; 47: 171.

28 Stanford JL. A vaccine for leprosy. Lepr Rev, 1979; 47: 87.

${ }^{29}$ Convit J, Aranzazu N, Ulrich M, Pinardi ME, Reyes O, Alvarado J. Immunotherapy with a mixture of Mycobacterium leprae and BCG in different forms of leprosy and in mitsuda negative contacts. Int J Lepr, 1982; 50: 415.

${ }^{30}$ Shepard CC, van Landingham RM, Walker LL, Ye S-Z. Comparison of the immunogenicity of vaccines prepared from viable Mycobacterium bovis $\mathrm{BCG}$, heat-killed Mycobacterium leprae, and a mixture of the two for normal and M. leprae-tolerant mice. Infect Immun, 1983; 40: 1096. 